\title{
How to Evaluate the Performance of Traction Battery for Electric Vehicles
}

\section{Short Communication}

Volume 2 Issue 2- 2021

\begin{abstract}
Author Details
Tianyi Ma*, Dapeng Xu and Fang Wang

Tianjin Key Laboratory of Evaluation Technology for Electric Vehicles, China Automotive Technology and Research Center Co., Ltd. (CATARC), Tianjin 300300, China

*Corresponding author

Tianyi Ma, Tianjin Key Laboratory of Evaluation Technology for Electric Vehicles, China Automotive Technology and Research Center Co., Ltd. (CATARC), Tianjin 300300, China
\end{abstract}

Article History

Received: March 15, 2021 Accepted: March 19, 2021 Published: March 22, 2021

\begin{abstract}
With the rapid development of new energy vehicle technology, how to test and evaluate the performance of a traction battery has become a key issue in the field of new energy vehicle testing technology. Starting from the technical requirements of vehicle traction battery, this mini review introduces the key contents of battery performance test and evaluation from vehicle level and battery level respectively. Furthermore, based on the actual vehicle demand, the influence of electricity quality and feedback current on the performance of battery is introduced, and the feasibility of multi-stage reuse of traction battery is discussed.
\end{abstract}

Keywords: Traction battery; Performance evaluation; Electricity quality; Feedback current; Multi-stage reuse.

\section{Introduction}

The development of electric transportation brings up with higher requirements for the energy density of lithium-ion batteries (LIBs). Higher technical barriers increased competition between producers while the profit decreased continuously $[1,2]$. In order to control the production cost and product quality accurately, the accurate evaluation of traction battery performance has received extensive attention around the industry. The basic parameters of a battery include performance parameters, safety parameters, durability parameters, accompany with cost, environmental protection and so on. However, if the current demand is to evaluate the comprehensive performance of the battery to set a performance level for the battery, battery parameters cannot be the only criteria to consider, the application scenarios should be included to the evaluation system [3,4]. From the perspective of comprehensive evaluation, excellent products are defined as the products suitable for this application scenario. To be specific, high speed, high range, low cost, easy to operate, these targets are the common goal of the world. Therefore, for the evaluation of traction battery, it is necessary to design an appropriate evaluation system related to the above aspects.

Test and evaluation are servicing for industry. At present, the trend of test and evaluation of electric vehicle and battery performance is closely related to the characteristics of industry and product. In recent years, the sales volume of electric vehicles has increased rapidly year by year, which has formed a complete industrial chain and technology chain and is gradually in the trend of cost slowing down. The product iteration of the new system is very fast, including the use of new materials for batteries, the use of silicon carbide for motors, the lightweight design of chassis and whole vehicle, and the system integration technology [5-7]. High integration is the trend of current industry, and high integration will also affect the change of our battery standards and test evaluation objects. For example, how to evaluate the battery on integrated chassis.

\section{Performance evaluation of traction battery}

The performance test and evaluation of traction battery need to be analyzed from multilevel of the battery, and there is a coupling relationship between different levels. The first is material system, how to optimize the material system to take into account the high specific capacity and high-rate characteristics to meet the requirements of fast charging and fast discharging. The craft of electrode, separator and electrolyte is also the key factors to determine the capacity and power characteristics of the battery [8-10]. On the other hand, it is indispensable to consider how to ensure a stable performance in the whole life of a battery. At the level of battery system, how to simulate the condition of high-power output and other actual conditions on the thermal management system and battery management system (BMS) identification through laboratory testing and analysis [11,12]. In addition, quality of electricity, vehicle strategy and other problems 
which are easily ignored will also bring additional impact on battery performance. In the battery manufacturing process, measures such as thickening the separator, electrolyte additives and so on to improve the rate performance or rate safety will reduce the energy density, that is to say, there is a certain contradiction between the performance and safety in technical route; this is not only a challenge for the industrialized material system, but also a similar contradiction for solid state batteries and lithium-sulfur based batteries [13-15]. How to meet the requirements of energy density and high power and safety is the key problem to improve the performance of traction batteries.

At the cell level, the battery performance test system mainly includes the following aspects. The first is the consistency among different cells, including the consistency of weight, open circuit voltage, internal resistance, and capacity. When the cells are modular, the effect of consistency on the battery system is very important, at which come up with a cask effect. The second is the temperature and rate characteristics, which determine the operating range of the battery to give full play of its capacity and life. The working conditions of vehicle are complex, different vehicle types, road conditions and temperature changes in four seasons have different requirements on the temperature characteristics and rate characteristics of battery $[16,17]$. The third is the power characteristic, which is also the key demand for the acceleration and climbing performance of electric vehicles. Some of the electric vehicles that have been used for several years may still have certain range after charging, but they can't provide sufficient impetus during the acceleration stage. That is the results of the power characteristics of the battery have deteriorated because of aging. The fourth, energy density, under different working conditions, is also the core index affecting the range. The last part is the characteristics of heat generation. Although the heat generation characteristics are not directly related to the performance of the battery, the battery with large heat generation has higher requirements on cooling system, which indirectly improves the overall technical requirements of the battery system and reduces the energy density $[18,19]$.

\section{Factors affecting the performance of traction battery in practice}

The battery performance impact mentioned above is mainly the influence of material system and working condition on the performance of the battery. In practical application of electric vehicles, there are two aspects of influence that need to be considered.

On one hand, is the quality of electricity. The current of DC charging is mixed with some AC, which is determined by the capability of power supply system. According to the current test data, the change of the frequency of $\mathrm{AC}$ when peak value of $\mathrm{AC}$ sine wave is fixed and the change of the peak value when frequency is fixed are not show linear change. However, if AC is doped, it will have a significant impact on the capacity. Quality of electricity in different cities and regions, different charging piles is different. Whether the long-term use of different power quality will have a significant impact on the aging of the battery is still testing for a long period.

On the other hand, is the energy feedback condition included in the real vehicle application. When electric vehicle is braking at a high speed, it may cause a large instantaneous current feedback to the battery, which is similar to a pulse charging condition, but the specific form of pulse is uncertain. According to the data collection results of the real vehicle, there are two main parameters, initial speed and deceleration, which affect the feedback current. Energy feedback brings additional working pressure to the actual application scenario of traction battery, which may not be too high. The new factory battery can be fully accepted. However, if considering whether the aging battery can bear the same pressure in the whole life span, it needs a long-term test and verification.
The electricity and feedback current need to be considered in the performance analysis of the whole life cycle. In fact, as long as the analysis of traction battery is extended to the whole life cycle, more factors should be considered, and these factors are coupling and interrelated, sometimes it is difficult to test and verify single variable. For the tester, not only need to consider various factors, but also to find the most important factors of these factors and establish a feasible test method. For the real vehicle application, the core evaluation index of battery life is still capacity energy, internal resistance power, heat generation and consistency. But in these parameters, the main factors to consider are the rate of change and the threshold value of the boundary.

The multistage reuse of traction battery also requires the establishment of a perfect performance test method. After the battery is retired from the electric vehicle, it still has about $70 \%$ of the remaining capacity. The batteries after retirement can continue to serve in low pressure conditions, such as base station, low-speed electric vehicle, etc. It involves the process of sorting and reorganization, which needs to consider consistency and cost. If the battery needs to be retired after service under low pressure, we can actually use the battery in some of the lower pressure scenarios, such as lighting, etc. But at this time, with the current technical level of battery products, it is not suitable to use them in groups. From the perspective of working conditions, when reusing and secondary reuse, it is needed to consider many factors, such as ratio, voltage platform, frequency of use, etc. Different reuse routes need to be considered for different batteries of different systems, which is a comprehensive problem of performance, safety, durability and cost.

\section{Conclusion}

In summary, the performance test and evaluation technology of traction battery should focus on the vehicle demand and establish the test method and evaluation index. Also, the impact of the whole life cycle on the battery should be fully considered. In the specific technical route of establishing test and evaluation methods, it is necessary to combine the test methods of materials, cells, system and vehicle to form a multilevel comprehensive evaluation system.

\section{References}

1. A Sureth, V Moll, J Nachtwei (2019) The golden rules of ecodriving? The effect of providing hybrid electric vehicle (HEV) drivers with a newly developed set of ecodriving-tips. Transportation Research Part F-Traffic Psychology and Behaviour 64: 565-581.

2. Y Wu, L Zhang (2017) Can the development of electric vehicles reduce the emission of air pollutants and greenhouse gases in developing countries? Transportation Research Part D-Transport And Environment 51: $129-145$

3. V Ruiz, A Pfrang, A Kriston (2018) A review of international abuse testing standards and regulations for lithium-ion batteries in electric and hybrid electric vehicles. Renewable \& Sustainable Energy Reviews 81: $1427-1452$.

4. A Ahmad, ZA Khan, MS Alam (2018) A Review of the Electric Vehicle Charging Techniques, Standards, Progression and Evolution of EV Technologies in Germany. Smart Science 6: 36-53.

5. M Li, J Lu, Z Chen (2018) 30 years of lithium冈ion batteries. Adv Mater 30: 1800561 .

6. CC Kwasieffah, T Rabczuk (2018) Dimensional analysis and modelling of energy density of lithium-ion battery. Journal of Energy Storage 18: 308-315.

7. H Hesse, M Schimpe, D Kucevic (2017) Lithium-ion battery storage for the grid-A review of stationary battery storage system design tailored for applications in modern power grids. Energies 10: 2107. 
8. YL Yin, Y Hu, SY Choe (2019) New fast charging method of lithium-ion batteries based on a reduced order electrochemical model considering side reaction. J Power Sources 423: 367-379.

9. YQ Wu, WX Wang, J Ming (2019) An Exploration of New Energy Storage System: High Energy Density, High Safety, and Fast Charging Lithium-Ion Battery. Adv Funct Mater 29: 7.

10. J Sieg, J Bandlow, T Mitsch (2019) Fast charging of an electric vehicle lithium-ion battery at the limit of the lithium deposition process. J Power Sources 427: 260-270.

11. MA Hannan, MH Lipu, A Hussain (2017) A review of lithium-ion battery state of charge estimation and management system in electric vehicle applications: Challenges and recommendations. Renewable and Sustainable Energy Reviews 78: 834-854.

12. Q Wang, B Jiang, B Li (2016) A critical review of thermal management models and solutions of lithium-ion batteries for the development of pure electric vehicles. Renewable and Sustainable Energy Reviews 64: 106-128.

13. JL Shi, N Ehteshami, JL Ma (2019) Improving the graphite/electrolyte interface in lithium-ion battery for fast charging and low temperature operation: Fluorosulfonyl isocyanate as electrolyte additive. J Power Sources 429: 67-74
14. M Frankenberger, M Singh, A Dinter (2019) Laminated Lithium-Ion Batteries with improved fast charging capability. J Electroanal Chem 837: 151-158.

15. SJ Zhang, R Zhao, JJ Gu A (2018) Numerical Study of Lithium-Ion Battery Fast Charging Behaviors. Ieee, New York.

16. A Kriston, I Adanouj, V Ruiz (2019) Quantification and simulation of thermal decomposition reactions of Li-ion battery materials by simultaneous thermal analysis coupled with gas analysis. J Power Sources 435.

17. M Mayur, SC DeCaluwe, BL Kee (2019) Modeling and simulation of the thermodynamics of lithium-ion battery intercalation materials in the open-source software Cantera. Electrochim Acta 323.

18. JN Zhang, L Zhang, FC Sun (2018) An Overview on Thermal Safety Issues of Lithium-ion Batteries for Electric Vehicle Application. Ieee Access 6: 23848-23863.

19. TQ Wu, HD Chen, QS Wang (2018) Comparison analysis on the thermal runaway of lithium-ion battery under two heating modes. J Hazard Mater 344: 733-741. 